\title{
Innovando en la enseñanza de las diferentes teorías que explican el enlace químico
}

\author{
MARCELA MARTínez TEJAdA \\ Departamento de Química Inorgánica \\ Universidad de Sevilla \\ leidy@us.es \\ ORCID: https://orcid.org/0000-0002-0471-5033 \\ D.O.I.: http://dx.doi.org/10.12795/JDU.2018.i01.69 \\ Pp.: $1230-1248$
}

\section{Resumen}

En la presente comunicación se desarrolla una experiencia de innovación docente en la explicación del enlace químico por diferentes teorías. El modelo pedagógico implementado se basó en la interpretación y discusión en grupo de las diferentes teorías que explican el enlace entre los átomos. Se partió de los conocimientos previos de los estudiantes para saber redirigir la enseñanza hacia aquellos contenidos de menor comprensión y dificultad para los alumnos. Estas ideas previas sirvieron para establecer conceptos especificos iniciales, para que a través de procesos pedagógicos inductivos y con la ayuda de las tecnologías de la comunicación y la información, fueran capaces de llegar al conocimiento del concepto final planteado. La experiencia fue gratificante y motivadora para los estudiantes y para la docente. Los alumnos fueron capaces de establecer relaciones entre el conocimiento previo, el adquirido y el desarrollado durante la experiencia. 
Palabras claves: Química General y Analítica, Grado en Farmacia, Docencia Universitaria, Experimentación Docente Universitaria, Enlace Químico.

\section{Contexto}

El proyecto de innovación docente presentado se llevó a cabo en la asignatura Química General y Analítica del primer año del Grado en Farmacia. Esta asignatura es obligatoria y es impartida por dos Dptos., el de Química Analítica de la Facultad de Farmacia y el de Química Inorgánica de la Facultad de Química. El modelo pedagógico se desarrolló para el bloque de Química General. Esta asignatura se imparte durante el primer cuatrimestre y los alumnos no poseen conocimientos previos más allá de lo aprendido durante el Bachillerato. El alumnado aún tiene percepción de E.S.O. y Bachillerato, es poco independiente y autónomo. La asignatura se imparte en un aula convencional en donde el profesor se ubica en una tarima y los alumnos se encuentran sentados en dos hileras de mesas alineadas fijas de 10 asientos por fila. El profesor cuenta con pizarra y proyector como ayuda para sus tareas docentes.

La inquietud de realizar un modelo metodológico diferente al modelo clásico de enseñanza direccional (profesor alumno), y que sirviera para motivar e interiorizar conceptos de una mejor manera, surge del poco interés que presenta esta asignatura para los estudiantes de primero de Farmacia. Muestra de ello es el incremento de suspensos todos los años.

Con el modelo metodológico planteado se pretende que el alumno participe de una manera activa en su aprendizaje. De esta manera no solo acepta y repite lo enseñado, sino que es capaz de interiorizar lo aprendido y puede ser capaz de aplicarlo y relacionarlo con diferentes situaciones que se le presenten. Finalmente se busca 
que el alumno sea capaz de resolver cualquier cuestión planteada de una manera más crítica. Más allá de la importancia de que los alumnos aprendan ejemplos concretos, el aprendizaje significativo estriba en la interiorización de nuevos conceptos, y en la capacidad de utilizarlos de manera correcta para llegar a establecer relaciones entre ellos, combinando procesos de razonamiento inductivos y deductivos.

\section{Diseño del Ciclo de Mejora}

Debido a la enorme magnitud de temas y materias que deben abordarse durante la obtención de un grado, la idea de afrontar cursos o partes de la asignatura que pueden ser menos atractivas, usando asociaciones de conceptos a través de preguntas y objetivos claros, se plantea como una forma innovadora de acercar el conocimiento a los alumnos.

Muchos estudios han demostrado la efectividad del aprendizaje activo en una amplia variedad de disciplinas científicas (Bain, 2007), en donde los estudiantes están en contacto los unos con los otros como compañeros de aprendizaje (Ford and Wargo, 2012), y el profesor se retira del centro del aula para dar participación activa a los estudiantes. Cobra gran importancia la retroalimentación en el proceso formativo, de forma que se involucre a los estudiantes a través de la evidencia y la discusión haciéndose importante la instrucción (Hattie, 2009). En este sentido el profesor plantea preguntas específicas a los alumnos y les motiva para que lleguen a las soluciones, así se sentirán obligados a cuestionar sus respuestas y a pensar más allá.

Con este tipo de enseñanza los estudiantes estarán envueltos en un amplio rango de actividades de clase, usando las herramientas de las tecnologías de la información y comunicación, lectura, escritura, discusión, explicación y 
resolución de problemas. La manera para lograr que la experiencia sea enriquecedora para los alumnos, es que el trabajo de intentar llegar a la respuesta correcta se haga en grupo. Usando su propio razonamiento pueden cambiar y encontrar errores y respuestas, separar sus ideas, y ponerlas juntas de manera diferente. Todo ello conduce a la comprensión y de ahí al conocimiento (Finkel, 2000). De esta forma los alumnos serán conscientes de la relación indisoluble y esencial entre la realidad externa al aula y lo que se estudia dentro de ella, y podrán comprender las aplicaciones y utilidades de la Química General en su día a día. Así se pretende que comprendan desde un punto de vista más crítico el objetivo de la química en el descubrimiento de verdades científicas, que la química es una ciencia experimental básica basada en el método científico y que se apoya en el conocimiento anterior para generar nuevos conocimientos. Y finalmente que la química busca el conocimiento de la naturaleza, y la aplicación de ese conocimiento a dar explicación a lo que les rodea.

\section{Mapa de contenidos}

El mapa de contenidos propuesto para el bloque de Química General y los temas 4, 5 y 6 previsto para $6 \mathrm{~h}$ de clase se muestra en la Figura 1.

Jornadas de Formación e Innovación Docente del Profesorado | № 1 (2018) Esta obra se distribuye con la licencia Creative Commons 


\begin{tabular}{|c|c|c|c|c|c|c|}
\hline $\begin{array}{l}\text { ¿Por qué se } \\
\text { enlazan los } \\
\text { atomos? }\end{array}$ & $\begin{array}{l}\text { ¿Cómose } \\
\text { enlazan los } \\
\text { átomos? }\end{array}$ & $\begin{array}{l}\text { ¿Qué mantiene } \\
\text { unidos los } \\
\text { atomos? }\end{array}$ & $\begin{array}{l}\text { ¿Cuáles son } \\
\text { los tipos de } \\
\text { enlace? }\end{array}$ & $\begin{array}{l}\text { ¿De qué depende } \\
\text { el tipo de de } \\
\text { enlace? }\end{array}$ & \begin{tabular}{|l} 
¿Cómo se relacionan el tipo \\
de enlace con las \\
propiedades periódicas?
\end{tabular} & $\begin{array}{l}\text { ¿Cómo se relacionan el tipo de } \\
\text { enlace y la geometría de las } \\
\text { moléculas? }\end{array}$ \\
\hline
\end{tabular}

CONCEPTOS INICIALES

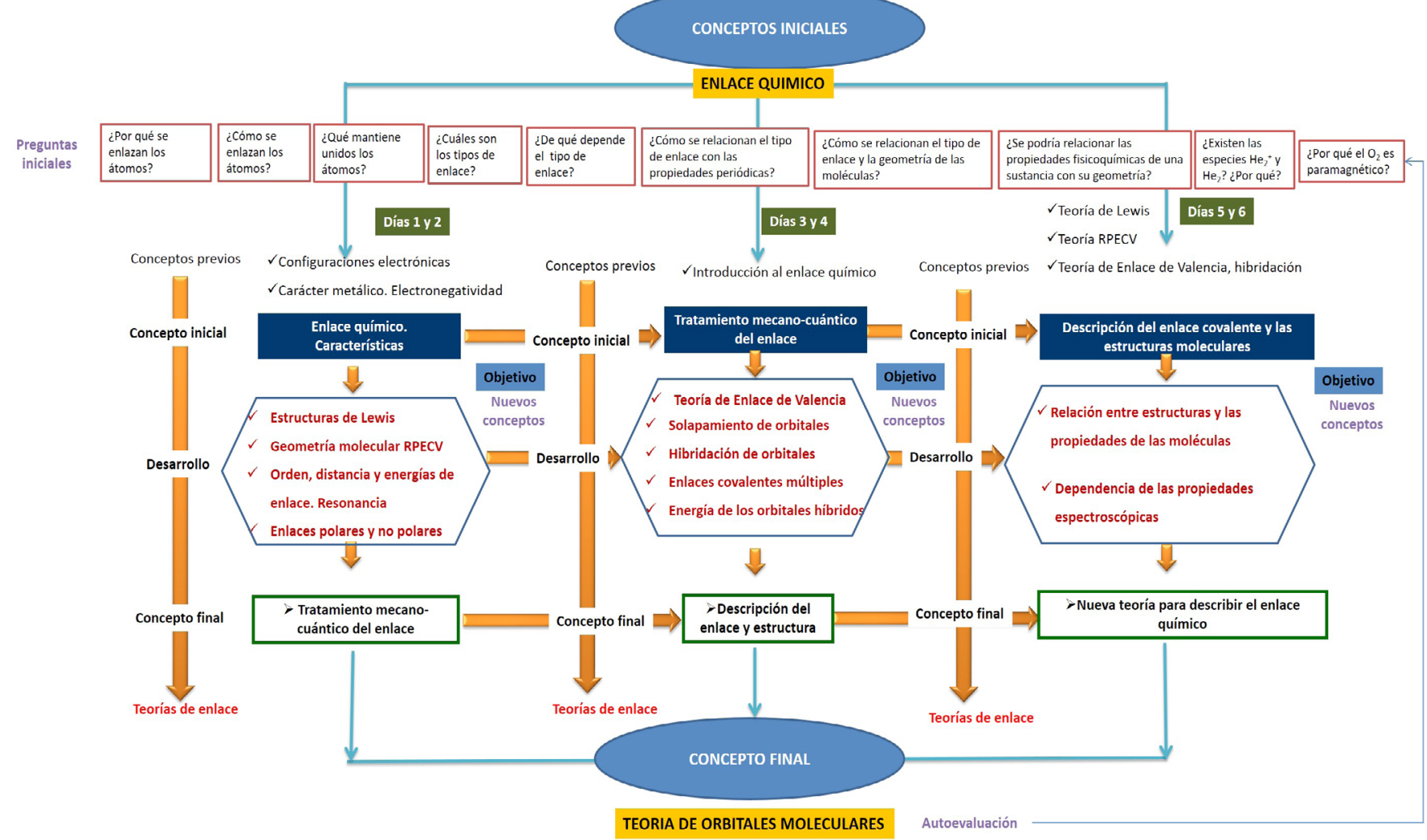

Figura 1. Mapa de contenidos para los conceptos impartidos durante $6 \mathrm{~h}$ de clase

Jornadas de Formación e Innovación Docente del Profesorado | № 1 (2018)

(c) E) Esta obra se distribuye con la licencia Creative Commons

(c) $)(-)$ Reconocimiento-NoComercial-SinObraDerivada

Internacional (CC BY-NC-ND 4.0.) 
El mapa de contenidos tiene como finalidad la comprensión de las diferentes teorías que explican el enlace químico. Está planteado para sesiones de 2 horas por cada tema, en donde se parte de un "concepto inicial" hasta llegar a un "concepto final" a través de diferentes subtemas que se encuentran en el apartado "Desarrollo". Estos subtemas se presentarán a los estudiantes en forma de objetivos a alcanzar en cada sesión. En el mapa de contenidos se incluyen además, 10 preguntas iniciales que ayudarán a recoger las ideas previas de los estudiantes acerca de los contenidos principales que se estudiarán durante el Ciclo de Mejora. Estas preguntas iniciales ayudarán a identificar los obstáculos y los conceptos erróneos, para luego redirigir la instrucción y la búsqueda de información para superarlos. La comparación entre las respuestas previas y las dadas tras el ciclo, servirá para evaluar, por un lado, el grado de comprensión de los estudiantes acerca de los conceptos fundamentales, y por otro, la capacidad de relacionarlos para dar explicación a fenómenos reales.

Las preguntas iniciales fueron: 1 . ¿Por qué se enlazan los átomos? 2. ¿Cómo se enlazan los átomos? 3. ¿Qué mantiene unidos los átomos? 4. ¿Cuáles son los tipos de enlace? 5. ¿De qué depende el tipo de enlace? 6. ¿Cómo se relacionan el tipo de enlace con las propiedades periódicas? 7. ¿Cómo se relacionan el tipo de enlace y la geometría de las moléculas? 8. ¿Se podrían relacionar las propiedades fisicoquímicas de una sustancia con su geometría? ¿Por qué? 9. ¿Existen las especies $\mathrm{He}_{2}{ }^{+}$y $\mathrm{He}_{2}$ ? ¿Por qué? 10. ¿Por qué el $\mathrm{O}_{2}$ es paramagnético?

\section{Modelo metodológico y secuencia de actividades}

El modelo metodológico seguido se muestra en la Figura 2. Con él se quiere estimular la curiosidad intelectual, la iniciativa y la creatividad, esto a su vez ayudará a los estudiantes a desarrollar la autonomía, la responsabilidad y la colaboración. De esta manera no solo aceptan y repiten lo enseñado, sino que son capaces de interiorizar lo aprendido para ser capaces de aplicarlo y relacionarlo con diferentes situaciones que se le presenten. El modelo se centra 
en la comprensión por parte de los alumnos de los conceptos más importantes que se tratarán en la clase a través de la búsqueda y explicación de objetivos, sumado a las discusiones/reflexiones con el profesor y con sus compañeros, para resolver las dudas generales. Con este modelo el alumno tendrá además la posibilidad no solo de conocer a los compañeros que se sientan al lado, sino también de compartir con él o con ellos la experiencia, dudas teóricas e impresiones de la actividad. Luego todas las ideas generadas se colocarán en común en forma de asamblea en donde el profesor será solo un moderador. El profesor dirigirá, re-construirá y corregirá las ideas erróneas obtenidas durante su proceso de búsqueda de información. Al finalizar el segundo ciclo de mejora se les regresará el test de preguntas previas a los estudiantes para que puedan autoevaluarse.

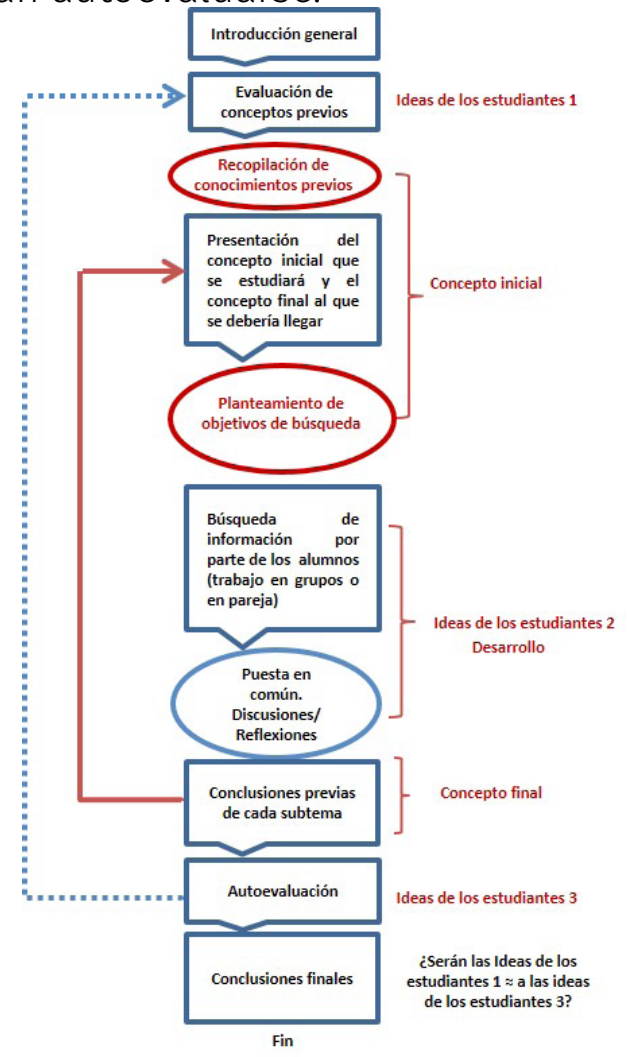

Figura 2. Modelo metodológico empleado.

Jornadas de Formación e Innovación Docente del Profesorado | № 1 (2018) Esta obra se distribuye con la licencia Creative Commons 

ciclo de Mejora.

Tabla 1: Secuencia de actividades del Ciclo Mejora (clases de $50 \mathrm{~min})$.

\begin{tabular}{|c|c|c|}
\hline $\begin{array}{l}\text { Tema } 4 \\
\text { Día } 1\end{array}$ & Día 2 & $\begin{array}{l}\text { Tema } 5 \\
\text { Día } 3\end{array}$ \\
\hline $\begin{array}{l}\mathbf{5} \text { min } \\
\text { Explicación de la me- } \\
\text { todología que se de- } \\
\text { sarrollará en las } \\
\text { próximas } 6 \text { clases. Re- } \\
\text { partir el test de cono- } \\
\text { cimientos previos. } \\
\mathbf{1 0 ~ m i n} \\
\text { Realización del test de } \\
\text { conocimientos previos. } \\
\text { Recogida del test. } \\
\mathbf{5} \text { min } \\
\text { Reorganización del } \\
\text { aula de clase for- } \\
\text { mando grupos de tres } \\
\text { o cuatro alumnos. Con } \\
\text { el mapa de contenidos } \\
\text { se hará la presenta- } \\
\text { ción del concepto del } \\
\text { día 1, y el concepto fi- } \\
\text { nal al que deben lle- } \\
\text { gar el día 2, y los } \\
\text { objetivos del día 1. } \\
\text { Concepto inicial: } \\
\text { Enlace químico. } \\
\text { Características. } \\
\text { Concepto final: Trata- } \\
\text { miento mecano-cuán- } \\
\text { tico del enlace. }\end{array}$ & $\begin{array}{l}\mathbf{5} \text { min } \\
\text { Resumen de las con- } \\
\text { clusiones del día } \\
\text { anterior. } \\
\text { Con el mapa concep- } \\
\text { tual, poner en situa- } \\
\text { ción los conceptos } \\
\text { iniciales y finales } \\
\text { planteados en el día } \\
\text { 1 y proponer el obje- } \\
\text { tivo del día 2: Poder } \\
\text { deducir la estructura } \\
\text { molecular, sabiendo } \\
\text { diferenciarla de la es- } \\
\text { tructura electrónica } \\
\mathbf{2 5} \text { min } \\
\text { Los alumnos con- } \\
\text { tinúan con la bús- } \\
\text { queda de información } \\
\text { del día para alcan- } \\
\text { zar el objetivo. En este } \\
\text { tiempo formarán sus } \\
\text { propias moléculas y } \\
\text { observarán la geome- } \\
\text { tría obtenida (apren- } \\
\text { der jugando). } \\
20 \text { min } \\
\text { Toda la información } \\
\text { obtenida desde los } \\
\text { alumnos se resumirá } \\
\text { y concretará, se co- } \\
\text { rregirán los errores } \\
\text { que se hayan podido } \\
\text { encontrar. }\end{array}$ & $\begin{array}{l}\mathbf{1 0} \text { min } \\
\text { Reorganización del } \\
\text { aula de clase for- } \\
\text { mando grupos de tres } \\
\text { o cuatro alumnos. } \\
\text { Resumen de las con- } \\
\text { clusiones de los dos } \\
\text { días anteriores. } \\
\text { Con base en el mapa } \\
\text { conceptual, se presen- } \\
\text { tarán los conceptos } \\
\text { iniciales y finales de } \\
\text { las dos próximas se- } \\
\text { siones (días } 3 \text { y 4) y la } \\
\text { presentación del obje- } \\
\text { tivo del día 3. } \\
\text { Concepto inicial: Trata- } \\
\text { miento mecano-cuán- } \\
\text { tico del enlace. } \\
\text { Concepto final: Des- } \\
\text { cripción del enlace y } \\
\text { la estructura } \\
\text { Objetivo del día 3: Co- } \\
\text { nocer las bases de la } \\
\text { Teoría de Enlace Va- } \\
\text { lencia y el concepto } \\
\text { de Orbitales Atómicos } \\
\text { Híbridos. } \\
\mathbf{2 0 ~ m i n ~} \\
\text { Búsqueda de informa- } \\
\text { ción por parte de los } \\
\text { alumnos. }\end{array}$ \\
\hline
\end{tabular}

Jornadas de Formación e Innovación Docente del Profesorado | № 1 (2018) Esta obra se distribuye con la licencia Creative Commons Reconocimiento-NoComercial-SinObraDerivada Internacional (CC BY-NC-ND 4.0.) 


\section{Objetivos del día 1:}

Conocer el por qué se

da un enlace químico

y la relación existente

entre la posición de

un elemento en la ta-

bla periódica y el tipo

de enlace. Saber es-

cribir la estructura de

Lewis de una molé-

cula. Comprender el

concepto resonancia y

las implicaciones que

tiene sobre concep-

tos como la distancia

de enlace y la energía

de enlace

\section{0 min}

Durante este tiempo

los alumnos usando

sus teléfonos, tabletas

y compartiendo con

los compañeros, bus-

carán la información

que crean adecuada

para alcanzar el objetivo del día.

\section{0 min}

Toda la información

obtenida se resumirá

y concretará, se corre-

girán los errores para

refinar la búsqueda y

\section{0 min}

Toda la información obtenida desde los alumnos se resumirá y concretará, se corregirán los errores que se hayan podido encontrar. 
Igual metodología que el día 2 pero con los objetivos del día

4. Justificar la aproximación del empleo de Orbitales Atómicos Híbridos para el átomo central de una especie poliatómica discreta en relación con su geometría y energía de enlace. Conocer y saber aplicar los diferentes tipos de Orbitales Atómicos Híbridos.
Igual metodología que el día 3 pero con los conceptos inicial y final de los días 5 y 6 , y con los objetivos de día 5

Concepto inicial: Descripción del enlace covalente y las estructuras moleculares

Concepto final: Nueva teoría para describir el enlace químico.

\section{Objetivo del día 5:}

Relacionar las estructuras y las propiedades de las moléculas, al igual que la dependencia de las propiedades espectroscópicas.
Igual metodología que el día 2 pero con los objetivos del día 6 . Conocer el método para obtener Orbitales Moleculares a partir de Combinaciones Lineales de Orbitales Atómicos. Conocer los requisitos de simetría, solapamiento y energía que han de cumplirse para aplicar dicho método a moléculas tipo $A_{2}$.

\section{$15 \mathrm{~min}$}

Una vez se hayan puesto en común todas las ideas, se regresará el test del día 1 para que cada alumno se autoevalúe.

Corrección del test mediante discusión/ reflexión con el alumnado. CONCLUSIONES Y FIN

Jornadas de Formación e Innovación Docente del Profesorado | № 1 (2018) Esta obra se distribuye con la licencia Creative Commons Reconocimiento-NoComercial-SinObraDerivada Internacional (CC BY-NC-ND 4.0.) 


\title{
Aplicación del Ciclo de Mejora
}

\author{
En la tabla 2 se recoge el relato resumido de las sesiones
}

Tabla 2: Relato de las 6 sesiones de clase.

\begin{tabular}{|c|c|c|}
\hline Día 1-Lunes & Día 2-Martes & Día 3-Lunes \\
\hline $\begin{array}{l}\text { Llego a clase y cuento } \\
65 \text { alumnos. Explico la } \\
\text { metodología. Entrego } \\
\text { el test de conocimien- } \\
\text { tos previos y lo recojo. } \\
\text { Reorganizo el aula y les } \\
\text { explico los conceptos } \\
\text { inicial y final y los obje- } \\
\text { tivos del día 1. Les digo } \\
\text { que tienen libertad de } \\
\text { buscar la información } \\
\text { con todas las herra- } \\
\text { mientas que dispongan } \\
\text { (Tablet, teléfono, orde- } \\
\text { nador) y con los com- } \\
\text { pañeros que tienen } \\
\text { al lado Durante este } \\
\text { tiempo respondo a sus } \\
\text { dudas de forma per- } \\
\text { sonalizada. El debate } \\
\text { / discusión es compli- } \\
\text { cado, es difícil que ha- } \\
\text { blan con orden y se } \\
\text { escuchen unos a otros, } \\
\text { pero acabamos conclu- } \\
\text { yendo definiciones con- } \\
\text { cretas de los conceptos } \\
\text { planteados. Uno de los } \\
\text { alumnos tomó la vo- } \\
\text { cería con reflexiones y } \\
\text { preguntas y respuestas } \\
\text { claras acerca del tema, } \\
\text { prácticamente pasó a } \\
\text { ser el moderador de } \\
\text { la situación, Sin em- } \\
\text { bargo tuve que interve- } \\
\text { nir en varias ocasiones } \\
\text { para aclarar mejor los } \\
\text { conceptos. }\end{array}$ & $\begin{array}{l}\text { Llego al aula y paso } \\
\text { lista, } 64 \text { alumnos. Re- } \\
\text { sumo las conclusiones } \\
\text { del día anterior. Anoto } \\
\text { en la pizarra el con- } \\
\text { cepto inicial y final de } \\
\text { los días 1 y 2, y el obje- } \\
\text { tivo del día 2. Recuerdo } \\
\text { que pueden pedir } \\
\text { ayuda al compañero } \\
\text { (s) que tienen al lado, } \\
\text { aparte del uso de todas } \\
\text { las herramientas de las } \\
\text { que dispongan. Da la } \\
\text { impresión que hay ma- } \\
\text { yor implicación en la } \\
\text { búsqueda de informa- } \\
\text { ción. Esta vez además } \\
\text { les doy algunas molé- } \\
\text { culas para que deter- } \\
\text { minen su geometría } \\
\text { usando modelos con } \\
\text { esferas y palillos, y dis- } \\
\text { frutan de la actividad. } \\
\text { Intento tener mejor } \\
\text { control del debate, pre- } \\
\text { gunto directamente sus } \\
\text { impresiones y relacio- } \\
\text { nes de los dos concep- } \\
\text { tos. Voy tomando nota } \\
\text { en la pizarra. Creo que } \\
\text { esta segunda actividad } \\
\text { los ha motivado aún } \\
\text { más. Al menos las res- } \\
\text { puestas y relaciones } \\
\text { dadas estuvieron mejor } \\
\text { resueltas que las de la } \\
\text { sesión anterior. }\end{array}$ & $\begin{array}{l}\text { Llego al aula y paso } \\
\text { lista, } 66 \text { alumnos. Re- } \\
\text { organizo el aula y le } \\
\text { explico los conceptos } \\
\text { iniciales y finales de } \\
\text { los días } 3 \text { y } 4 \text {. Tras ello } \\
\text { comienzan la búsqueda } \\
\text { de información sin de- } \\
\text { cirles el objetivo del } \\
\text { día } 4 \text {. Esta vez observo } \\
\text { que ya debaten y discu- } \\
\text { ten entre ellos. Amplio } \\
\text { el tiempo de discusión. } \\
\text { Durante este tiempo } \\
\text { ellos por si mismos son } \\
\text { capaces de formular el } \\
\text { objetivo del día } 3 \text {. Con } \\
\text { base en él, reoriento la } \\
\text { discusión hacia la me- } \\
\text { jor comprensión del } \\
\text { concepto inicial. }\end{array}$ \\
\hline
\end{tabular}


Día 4-Martes

Llego al aula y paso

lista, 62 alumnos. Anoto

en la pizarra el con-

cepto inicial y final de

los días 3 y 4 . Intro-

duzco el objetivo del

día. De nuevo de forma

totalmente autónoma

se organizan y comien-

zan con la búsqueda

de la información. El

debate y discusión es

participativo y se nota

una implicación alta. Al

final resolvemos en pi-

zarra algunos ejemplos

prácticos para la mejor

comprensión del tema.

Dos alumnos salen vo-

luntarios a la pizarra.

\section{Día 5-Lunes}

Llego al aula y paso

lista, 45 alumnos. Re-

organizo el aula y le

explico los concep-

tos iniciales y finales

de los días 5 y 6 . De-

baten y discuten en-

tre ellos, sin embargo,

se notan preocupados

por un parcial que tie-

nen de otra asignatura.

El debate es poco par-

ticipativo. Voy tomando

nota en la pizarra. Las

respuestas y relacio-

nes dadas por parte de

los alumnos estuvieron

bien resueltas sin em-

bargo con poca partici-

pación. Esta vez no se

notan tranquilos.

\section{Día 6-Martes}

Llego al aula y paso

lista, 66 alumnos. Re-

sumo las conclusiones.

Con una serie de molé-

culas $\mathrm{A}_{2}$ les pido obte-

ner los OM a partir de

sus orbitales atómicos.

Se implican bastante

en la búsqueda de in-

formación. Luego les

regreso el test inicial

e insisto que lo hagan

con bolígrafo. Luego

con un lápiz lo corre-

gimos entre todos, re-

cojo el test. Finalizo

con reflexiones y las

conclusiones a las que

llegamos en las 6 se-

siones. Creo que la ac-

tividad en general les

ha gustado. FIN.

\section{Evaluación del aprendizaje de los estudiantes}

Es difícil saber qué aprenden y cómo lo hacen los estudiantes. El uso de cuestionarios, entrevistas, observación y combinaciones de ellas de una manera adecuada, facilita identificar los obstáculos que se presentan en el aprendizaje (Porlán, 2017). Tras el cuestionar se debe clasificar la información para conocer niveles y obstáculos. Para la evaluación en este segundo ciclo mejora, se utilizó un test de conocimientos previos. Tras las 6 sesiones este test fue de nuevo realizado por los alumnos para observar su avance. La tabla 3 muestra los resultados de las respuestas dadas a algunas de las preguntas usando una escalera de conocimientos. Las preguntas se seleccionaron con base en los contenidos teóricos y prácticos que mostraron mayor dificultad y que son fundamentales para comprender los siguientes. El nivel 4 es el escalón más alto y el nivel 1 es el 
más bajo. En pre- y pos-test se exponen los \% de alumnos que han dado el concepto mostrado como respuesta. Al final de cada pregunta se muestra el obstáculo general inicial observado para superar durante el ciclo de mejora.

Tabla 3: Escaleras de conocimientos en el pre-test y en el pos-test.

\begin{tabular}{|c|c|c|c|}
\hline Respuestas & Pre-test & Pos-test & Nivel \\
\hline $\begin{array}{l}\text { Concepto general: Dar } \\
\text { estabilidad. }\end{array}$ & $56 \%$ & $72 \%$ & 4 \\
\hline $\begin{array}{l}\text { Concepto cotidiano: Comple- } \\
\text { tar octete. }\end{array}$ & $16 \%$ & $20 \%$ & 3 \\
\hline $\begin{array}{l}\text { Otros conceptos: diferencia } \\
\text { de cargas, } \\
\text { mantener estructuras, } \\
\text { mismo número de electro- } \\
\text { nes, formar compuestos. }\end{array}$ & $22 \%$ & $8 \%$ & 2 \\
\hline No sabe/no contesta. & $6 \%$ & $0 \%$ & 1 \\
\hline
\end{tabular}

- Pregunta 2: ¿Cómo se enlazan los átomos?

\begin{tabular}{|l|c|c|c|}
\hline \multicolumn{1}{|c|}{ Respuestas } & Pre-test & Pos-test & Nivel \\
\hline $\begin{array}{l}\text { Concepto general: Compar- } \\
\text { tición de electrones/en- } \\
\text { laces químicos (fuerzas } \\
\text { intramoleculares). }\end{array}$ & $43 \%$ & $50 \%$ & $\mathbf{4}$ \\
\hline $\begin{array}{l}\text { Concepto cotidiano: Ce- } \\
\text { diendo electrones. }\end{array}$ & $33 \%$ & $40 \%$ & $\mathbf{3}$ \\
\hline $\begin{array}{l}\text { Otros conceptos: Dar estabi- } \\
\text { lidad, fuerzas intermolecula- } \\
\text { res, tipo de enlace. }\end{array}$ & $16 \%$ & $10 \%$ & $\mathbf{2}$ \\
\hline No sabe/no contesta. & $8 \%$ & $0 \%$ & $\mathbf{1}$ \\
\hline \multicolumn{2}{|c|}{ Obstáculo general inicial: Concepto de enlace y sus implicaciones. } \\
\hline
\end{tabular}

Jornadas de Formación e Innovación Docente del Profesorado | № 1 (2018) Esta obra se distribuye con la licencia Creative Commons Reconocimiento-NoComercial-SinObraDerivada Internacional (CC BY-NC-ND 4.0.) 
- Pregunta 3: ¿Qué mantiene unidos los átomos?

\begin{tabular}{|l|c|c|c|}
\hline \multicolumn{1}{|c|}{ Respuestas } & Pre-test & Pos-test & Nivel \\
\hline $\begin{array}{l}\text { Concepto general: Fuerzas de } \\
\text { atracción /electronegatividad } \\
\text { diferente. }\end{array}$ & $30 \%$ & $65 \%$ & $\mathbf{4}$ \\
\hline $\begin{array}{l}\text { Concepto cotidiano: Los } \\
\text { enlaces. }\end{array}$ & $28 \%$ & $15 \%$ & $\mathbf{3}$ \\
\hline $\begin{array}{l}\text { Otros conceptos: Diferencia } \\
\text { de energía, carga nuclear, los } \\
\text { electrones. }\end{array}$ & $26 \%$ & $20 \%$ & $\mathbf{2}$ \\
\hline \begin{tabular}{l} 
No sabe/no contesta. \\
\hline $\begin{array}{l}\text { Obstáculo general inicial: Electronegatividad/tipo de enlace/estabilidad/ } \\
\text { carga nuclear efectiva. }\end{array}$
\end{tabular} \\
\hline
\end{tabular}

- Pregunta 5: ¿De qué depende el tipo de enlace?

\begin{tabular}{|l|c|c|c|}
\hline \multicolumn{1}{|c|}{ Respuestas } & Pre-test & Pos-test & Nivel \\
\hline $\begin{array}{l}\text { Concepto general: Na- } \\
\text { turaleza de los átomos/ } \\
\text { electronegatividad. }\end{array}$ & $47 \%$ & $87 \%$ & $\mathbf{4}$ \\
\hline $\begin{array}{l}\text { Concepto cotidiano: Carácter } \\
\text { metálico. }\end{array}$ & $20 \%$ & $13 \%$ & $\mathbf{3}$ \\
\hline $\begin{array}{l}\text { Otros conceptos: Teo- } \\
\text { ría de Lewis, cantidad de } \\
\text { electrones. }\end{array}$ & $16 \%$ & $0 \%$ & $\mathbf{2}$ \\
\hline \begin{tabular}{l} 
No sabe/no contesta. \\
\hline $\begin{array}{l}\text { Obstáculo general inicial: Electronegatividad/tipo de enlace/estabilidad/ } \\
\text { carga nuclear efectiva. }\end{array}$
\end{tabular} \\
\hline
\end{tabular}

Jornadas de Formación e Innovación Docente del Profesorado | № 1 (2018) Esta obra se distribuye con la licencia Creative Commons Reconocimiento-NoComercial-SinObraDerivada Internacional (CC BY-NC-ND 4.0.) 
- Pregunta 6: ¿Cómo se relacionan el tipo de enlace con las propiedades periódicas?

\begin{tabular}{|l|c|c|c|}
\hline \multicolumn{1}{|c|}{ Respuestas } & Pre-test & Pos-test & Nivel \\
\hline $\begin{array}{l}\text { Concepto general: Carácter } \\
\text { metálico y no metálico del } \\
\text { enlace. }\end{array}$ & $37 \%$ & $67 \%$ & 4 \\
\hline $\begin{array}{l}\text { Concepto cotidiano: Con } \\
\text { la posición en la tabla } \\
\text { periódica. }\end{array}$ & $32 \%$ & $10 \%$ & $\mathbf{3}$ \\
\hline $\begin{array}{l}\text { Otros conceptos: Con la con- } \\
\text { figuración electrónica, con } \\
\text { la electronegatividad, con la } \\
\text { afinidad electrónica. }\end{array}$ & $26 \%$ & $23 \%$ & $\mathbf{2}$ \\
\hline \begin{tabular}{l} 
No sabe/no contesta \\
\hline $\begin{array}{l}\text { Obstáculo general inicial: Carácter metálico/no metálico/apantallamiento/ } \\
\text { penetración del orbital. }\end{array}$
\end{tabular} \\
\hline
\end{tabular}

- Pregunta 7: ¿Cómo se relacionan el tipo de enlace y la geometría de las moléculas?

\begin{tabular}{|c|c|c|c|}
\hline Respuestas & Pre-test & Pos-test & Nivel \\
\hline $\begin{array}{l}\text { Concepto general: Repul- } \\
\text { siones interelectrónicas/ } \\
\text { VSPER }\end{array}$ & $24 \%$ & $55 \%$ & 4 \\
\hline $\begin{array}{l}\text { Concepto cotidiano: Por el } \\
\text { número de electrones que } \\
\text { comparten. }\end{array}$ & $16 \%$ & $17 \%$ & 3 \\
\hline $\begin{array}{l}\text { Otros conceptos: Es- } \\
\text { tructura de Lewis, por la } \\
\text { electronegatividad, } \\
\text { fuerzas del enlace, repulsio- } \\
\text { nes electromagnéticas. }\end{array}$ & $41 \%$ & $28 \%$ & 2 \\
\hline No sabe/no contesta & $19 \%$ & $0 \%$ & 1 \\
\hline
\end{tabular}

Jornadas de Formación e Innovación Docente del Profesorado | № 1 (2018) Esta obra se distribuye con la licencia Creative Commons Reconocimiento-NoComercial-SinObraDerivada Internacional (CC BY-NC-ND 4.0.) 
- Pregunta 8: ¿Se podrían relacionar las prop. Fisicoquím. de una sustancia con su geometría?

\begin{tabular}{|c|c|c|c|}
\hline Respuestas & Pre-test & Pos-test & Nivel \\
\hline $\begin{array}{l}\text { Concepto general: Por su } \\
\text { polaridad/carácter polar del } \\
\text { enlace. }\end{array}$ & $33 \%$ & $85 \%$ & 4 \\
\hline $\begin{array}{l}\text { Concepto cotidiano: : Si, sin } \\
\text { dar explicación }\end{array}$ & $25 \%$ & $0 \%$ & 3 \\
\hline $\begin{array}{l}\text { Otros conceptos: Por la po- } \\
\text { sición espacial del grupo de } \\
\text { electrones, por el tipo de } \\
\text { enlace que forman, por re- } \\
\text { pulsiones interlectrónicas. }\end{array}$ & $17 \%$ & $15 \%$ & 2 \\
\hline No sabe/no contesta & $25 \%$ & $0 \%$ & 1 \\
\hline
\end{tabular}

Tras el análisis de las escaleras inicial y final, se observa que la gran mayoría de estudiantes lograron superar los obstáculos generales para comprender y relacionar los contenidos más importantes de los temas estudiados. Llegaron a comprender las diferentes teorías que explican el enlace químico, sus bases teóricas y sus relaciones, para explicar desde un punto de vista más crítico, el cómo, el por qué y el para qué se unen los átomos. Como concepto general de difícil comprensión, resalto la polaridad de las moléculas y de los enlaces, y su relación con la geometría (VSPER) y con las propiedades fisicoquímicas de las sustancias. Este concepto será necesario trabajarlo en mayor profundidad en el curso siguiente de Química Inorgánica de segundo cuatrimestre.

\section{Evaluación del diseño puesto en práctica}

Poco a poco durante los días del ciclo de mejora los alumnos se sintieron más cómodos con la metodología, 
aprovechando al máximo cada sesión. Llegaron a interiorizar la gran mayoría de los conceptos y a saber relacionarlos para llegar a los objetivos planteados cada día. Esto hizo la experiencia de docencia muy gratificante ya que se aumentó de una manera exponencial la participación de los alumnos, siendo muchas veces moderadores y guías de la discusión.

Finkel (2000) propone 4 elementos claves para que este tipo de enseñanza sea efectiva: personas con quien hablar, preguntas, profesor(a) a quien acudir y conclusiones. Monereo también concibe la enseñanza como la vinculación a la realidad del alumnado, con un conjunto de actividades instruccionales y de evaluación secuenciadas y conectadas en el tiempo, que plantean al alumnado problemas y conflictos a resolver (Monereo, 2009). Así el tipo de enseñanza innovadora debe ser realista, ser relevante (los aprendizajes deben tener sentido y la competencia desarrollada debe ser de utilidad al alumnado); ser constructivista (plantear actividades para captar el proceso de construcción que desarrolla el alumnado con el propósito de ofrecer ayudas ajustadas a los problemas que puedan ir apareciendo); ser socializante (apropiación de los discursos, signos o valores de identidad de la comunidad profesional en la que el alumnado va a integrarse). El profesor además debe captar el interés del alumno, construyendo ideas que lo lleven a solucionar el problema de partida planteado, sumando factores para que el aprendizaje sea gradual, diseñando entornos para que el estudiante se sienta seguro de poder llegar a la respuesta (Finkel, 2000). Por tanto es importante brindarles objetivos y un camino para llegar a ellos. Es decir darles a los estudiantes un comienzo, un medio y un fin. Con todo esto se busca que los alumnos progresen intelectualmente guiando la situación, de manera que cada alumno sea testigo de sus avances, conozca la materia de estudio, a sus compañeros, y se centre en la información dada, en las preguntas y en la instrucción para dar con la respuesta. Es una manera 
diferente de poner el estudiante a trabajar y que sean ellos aprendiendo, no solo el profesor enseñando.

En cuanto a los aspectos que han funcionado bien y que quiero mantener en mi práctica docente quiero destacar el uso de las nuevas tecnologías en la clase. Éstas les ayudaron a ser totalmente autónomos y a interesarse por los contenidos, llegando a ellos a través de relaciones de los conceptos más importantes. El planteamiento de objetivos de aprendizaje cada día y el uso de conceptos iniciales y finales para guiar la búsqueda de información, generaron mayor interés, además de fomentar el trabajo en equipo y la reflexión crítica sobre los contenidos. Simultáneamente el debate de las ideas entre todos, les ayudó a que se sintieran en un ambiente más cómodo rodeado de sus compañeros, dando forma a sus ideas, con mayor motivación. Pensar juntos en voz alta fue una manera de que el grupo progresara, dando forma entre todos a los contenidos más importantes y a sus relaciones. Todo ello despertó el interés y estudio por los contenidos tratados.

Como dijimos antes, se detectaron además contenidos de difícil comprensión sobre los se debe profundizar en el segundo cuatrimestre de Química Inorgánica, empleando material complementario de apoyo, y otros conceptos y objetivos adicionales.

\section{Conclusiones finales}

La metodología empleada favoreció que los alumnos progresaran intelectualmente guiando la situación, de manera que fueron testigos de sus avances, y llegaron a conocer la materia de estudio, a sus compañeros, y se centraron en comprender los conceptos. Se activó la curiosidad de los alumnos y se fortaleció la interacción y el desarrollo personal. El modelo de evaluación a través de escalera de conocimientos, ayudó a informarme y a poder tomar decisiones del mapa, metodología, actividades y de la propia evaluación para redirigir y reestructurar la enseñanza. Todo 
esto fue fruto del buen uso de las tecnologías de la información y a las instrucciones dadas, a través de objetivos y conceptos de búsqueda. Así los alumnos, en su gran mayoría, llegaron a relacionar y comprender los contenidos estudiados más importantes. Todo esto hizo la experiencia de innovación docente en la enseñanza de las diferentes teorías que explican el enlace químico, muy motivadora y gratificante. Para los próximos cursos deseo ampliar el ciclo de mejora a más contenidos. De esta forma podré recopilar más experiencias y tener un enfoque más global para diseñar más experiencias que verdaderamente enseñen.

\section{Referencias bibliográficas}

Bain K, (2007). Lo que hacen los mejores profesores universitarios. Universidad de Valencia.

Finkel, D. (2000). Dar clase con la boca cerrada. Universidad de Valencia.

Ford, M. J. y Wargo B. M. (2012). Dialogic Framing of Scientific Content for Conceptual and Epistemic Understanding. Science Education 96 (3).

Hattie, J. (2009). Visible Learning a Synthesis of over 800 Meta-Analyses Relating to Achievement. London. Routledge.

Monereo, C. (2009). PISA como excusa. Repensar la evaluación para cambiar la enseñanza. Barcelona. Grao.

Porlán R (2017). Enseñanza universitaria. Cómo mejorarla. Morata, Universidad de Sevilla.

Jornadas de Formación e Innovación Docente del Profesorado | № 1 (2018) Esta obra se distribuye con la licencia Creative Commons 